\title{
A NEW APPROACH TO PROBLEMS IN TWO DIMENSIONAL FLOW*
}

\author{
BY \\ MONROE H. MARTIN \\ University of Maryland
}

1. Introduction. The dynamical equations and the equation of continuity

$$
\rho\left(u_{x} u+u_{y} v\right)+p_{x}=0, \quad \rho\left(v_{x} u+v_{y} v\right)+p_{\nu}=0, \quad(\rho u)_{x}+(\rho v)_{\nu}=0,
$$

for the velocity components $u, v$, the density $\rho$ and the pressure $p$ in the steady twodimensional flow of a fluid, subject to no external forces, constitute an underdetermined system of three partial differential equations for four unknown functions

$$
u=u(x, y), \quad v=v(x, y), \quad \rho=\rho(x, y) \neq 0, \quad p=p(x, y),
$$

of the independent variables $x, y$.

To make the problem determinate, an additional relation may be prescribed, as is frequently done when it is assumed the density $\rho$ is a function of the pressure $p$. In this paper we prefer, at the outset, to deal with the underdetermined problem (1), reserving the right, however, to make the problem a determinate one whenever it is convenient to do so.

A novel feature in the investigation is the use of the stream function $\psi$ and the pressure $p$ as independent variables. With this device, the integration of (1), barring exceptional cases, ${ }^{1}$ is equivalent to the integration of a system

$$
\xi_{\psi}^{2}+\eta_{\psi}^{2}=q^{2}, \quad \xi_{\psi} \xi_{p p}+\eta_{\psi} \eta_{p p}=0, \quad q=q(\psi, p),
$$

of two partial differential equations for two unknown functions $\xi=\xi(\psi, p), \eta=\eta(\psi, p)$. Corresponding to the indeterminacy in (1), the fluid velocity $q=q(\psi, p)$ in (3) is an arbitrary function ${ }^{2}$ whose specification characterizes the system (3).

Once a solution of (3) has been found, the flow is presented parametrically by

$$
\begin{array}{lll}
x=-\eta_{p}(\psi, p), & u=\xi_{\psi}(\psi, p), & \bar{\xi}=\xi-p \xi_{p}, \\
y=\xi_{p}(\psi, p), & v=\eta_{\psi}(\psi, p), & \bar{\eta}=\eta-p \eta_{p} .
\end{array}
$$

For $\psi=$ const. the equations in the first and second columns constitute parametric equations respectively for the stream lines in the physical plane and their hodographs in the hodograph plane, the pressure $p$ serving for parameter. In the third column $\bar{\xi}, \bar{\eta}$ denote lift and drag functions.

Provided the flow does not have a degenerate hodograph, the variables $\psi, p$ may be eliminated from (4), and the usual transformation from the $(u, v)$-plane (hodograph plane) to the $(x, y)$-plane (physical plane) is obtained.

*Received March 24, 1949. Presented September 9, 1948 at the Madison meeting of the American Mathematical Society. This investigation was carried out under project NOL 139(Tm5-1) "Rotational Gas Flows" of the Naval Ordnance Laboratory, White Oak, Silver Springs 19, Maryland.

'See Theorem 1 in Sec. 2.

2The phrase "arbitrary function" is used in a restricted sense throughout the paper to designate a function subject to such continuity restrictions as may be necessary to insure the validity of any argument in which it enters, but which is otherwise entirely arbitrary. 
The form of equation (3) is invariant under the change of parameter

$$
\psi=\psi\left(\psi^{*}\right)
$$

a property that leads immediately to the Munk-Prim substitution principle ${ }^{3}$ for the special case of two dimensional flows.

By eliminating one of the unknown functions in (3), a Monge-Ampère partial differential equation is obtained for the other. In view of the symmetry of (3), the unknowns $\xi, \eta$ satisfy the same Monge-Ampère equation.

The application of the Cauchy-Kowalewsky existence and uniqueness theorem to this Monge-Ampère equation for a prescribed regular analytic function $q^{2}(\psi, p)$ leads immediately to an existence and uniqueness theorem for flows having a preassigned analytic pressure and velocity distribution along a prescribed analytic arc $C$. The functions $\xi, \eta$ in (4) are presented by power series

$$
\xi=h_{0}+h_{1}\left(\psi-\psi_{0}\right)+\cdots, \quad \eta=k_{n}+k_{1}\left(\psi-\psi_{0}\right)+\cdots,
$$

in which the $h, k$ are regular analytic functions of $p$ which are readily calculated from the initial data by elementary algebraic operations and differentiations.

A necessary and sufficient condition for (3) to have a solution (5) linear in $\psi-\psi_{0}$ is that the flow be irrotational. For a solution of this type the flow defined by (4) is the well known Prandtl-Meyer flow, ${ }^{4}$ and a simple geometric construction results for the stream lines.

The study of flows for which $\xi, \eta$ are polynomials in $\psi-\psi_{0}$ offers an attractive problem for investigation left untouched in the present paper and should lead to interesting examples of rotational gas flows.

If the drag function $\bar{\xi}$ (lift function $\bar{\eta}$ ) defined in (4) be introduced in place of $\xi(\eta)$ as unknown function, and $\psi, y(\psi, x)$ taken for independent variables by an Ampère contact transformation, the Monge-Ampère equation for $\xi(\eta)$ is replaced by a quasilinear equation for $\bar{\xi}(\bar{\eta})$. This leads to a general formulation of the method followed by Kiebel $^{5}$ in his investigation of rotational flows. Unfortunately, a simple parametric representation for the flow, analogous to (4), no longer appears to be available.

In the final paragraph the treatment of one-dimensional unsteady flows is reduced to a minor formal modification of the method followed in the study of two-dimensional steady flows.

2. The equations of motion. As is well known, the system (1) can be written in the alternative form

$$
\left(p+\rho u^{2}\right)_{x}+(\rho u v)_{y}=0, \quad(\rho u v)_{x}+\left(p+\rho v^{2}\right)_{\nu}=0, \quad(\rho u)_{x}+(\rho v)_{\nu}=0,
$$

${ }^{3}$ M. M. Munk and R. C. Prim, On the multiplicity of steady gas flows having the same streamline pattern. Proc. Nat. Ac. Sci., 33, 137-141 (1947). The principle is established by Munk and Prim for three dimensional flows.

${ }^{4} \mathrm{~T}$. Meyer, Ueber zweidimensionale Bewegungsvorgaenge in einem Gas, das mit Ueberschallgeschwindigkeit stroemt, Dissertation, Goettingen (1908) or Forschungsheft 62, V.D.I., Berlin (1908), pp. 31-67.

${ }^{5} \mathrm{I}$. A. Kiebel, An example of an exact solution of a two-dimensional rotational flow in gas dynamics (Russian). Prikl. Mat. Mekh. 11, 193-198 (1947). English translation by E. Rabkin, Technical Translation No. TT-35, National Research Council of Canada, Division of Mechanical Engineering. Ottawa (1947). 
from which it follows ${ }^{6}$ that, given a solution (2) of (1), there exist three functions $\xi, \bar{\eta}, \psi$ of $x, y$ such that $u, v, \rho, p, \bar{\xi}, \bar{\eta}, \psi$ constitute a solution to the system of Pfaff

$d \bar{\xi}=\rho u v d x-\left(p+\rho u^{2}\right) d y, d \bar{\eta}=\left(p+\rho v^{2}\right) d x-\rho u v d y, d \psi=\rho v d x-\rho u d y$.

The variable $\psi$ is, of course, the stream function, and to lend the variables $\bar{\xi}, \bar{\eta}$ physical significance, we write (6) in the form

$$
d \bar{\xi}=u d \psi-p d y, \quad d \bar{\eta}=v d \psi+p d x, \quad d \psi=\rho v d x-\rho u d y .
$$

Along a streamline

$$
d \bar{\xi}=-p d y, \quad d \bar{\eta}=p d x, \quad d \psi=0,
$$

from which the physical meaning of $\bar{\xi}, \bar{\eta}$ is apparent. The difference between the values of $\bar{\xi}$ at two points on a streamline forming the boundary of a solid immersed in the fluid equals the horizontal component of the force experienced by the body due to fluid pressure along the intervening arc. Since $\bar{\eta}$ may be interpreted as the vertical component of this force, the functions $\bar{\xi}, \bar{\eta}$ are frequently referred ${ }^{6}$ to as the drag and lift functions, respectively.

If we introduce auxiliary functions

$$
\xi=\bar{\xi}+p y, \quad \eta=\bar{\eta}-p x,
$$

of $x, y$, the system of Pfaff (6) becomes

$$
d \xi=u d \psi+y d p, \quad d \eta=v d \psi-x d p, \quad d \psi=\rho v d x-\rho u d y,
$$

which suggests that $\psi, p$ be taken as independent variables. This restricts us to those solutions (2) of (1) for which no functional relation exists between the functions $\psi, p$ of $x, y$. In other words we rule out at this point those flows for which $p=p(\psi)$, that is, flows for which the streamlines are isobars. Such flows form a very restricted class which will be studied in Sec. 3 .

With the introduction of $\psi, p$ as independent variables in place of $x, y$, the functions $\xi, \eta, \rho$ of $x, y$ become functions of $\psi, p$

$$
\xi=\xi(\psi, p), \quad \eta=\eta(\psi, p), \quad \rho=\rho(\psi, p) \neq 0,
$$

with $x, y, u, v, \bar{\xi}, \bar{\eta}$ given as functions of $\psi, p$ by (4). Substituting for $x, y, u, v$ from (4) in the last equation of (9) and remembering that $\psi, p$ are independent variables, we find the following conditions on the three functions (10)

$$
\xi_{\psi} \xi_{\psi p}+\eta_{\psi} \eta_{\psi p}+\rho^{-1}=0, \quad \xi_{\psi} \xi_{p p}+\eta_{\psi} \eta_{p p}=0 .
$$

Corresponding to a solution (2) of the underdetermined system (1), we are led by the above considerations to a solution (10) of the underdetermined system (11).

Let us consider the converse problem. Given a solution (10) of the underdetermined system (11) can a solution (2) of the underdetermined system (1) be constructed?

${ }^{6}$ See, for example, Bateman, H., The lift and drag functions for an elastic fluid in two-dimensional irrotational flow. Proc. Nat. Ac. Sci., 24, 246-251 (1938). The notation in this paper differs from ours, with Bateman writing $-\psi,-X,-Y$ for what we have designated by $\psi, \bar{\xi}, \bar{\eta}$. 
The underdetermined system (11) may be regarded as a determinate system of two partial differential equations for two unknown functions $\xi, \eta$, the function

$$
\rho=\rho(\psi, p) \neq 0,
$$

being regarded as an arbitrary function whose specification characterizes the system.

From this standpoint, the converse problem is formulated as follows. The function (12) being specified, given a solution $\xi, \eta$ of the determinate system (11), can a solution (2) of the underdetermined system (1) be constructed?

If we assume that

$$
\frac{\partial(x, y)}{\partial(\psi, \rho)}=\xi_{\psi p} \eta_{p p}-\eta_{\psi p} \xi_{p p}=J \neq 0,
$$

the equations in the first column of (4) determine $\psi, p$ as functions of $x, y$. Substitution for $\psi, p$ in the equations of the second column, and in (12), then yields $u, v, \rho$ as functions of $x, y$. We shall now show that the four functions (2) of $x, y$ so obtained constitute a solution of (1).

For this we need the following formulae derivable from (4):

$$
\begin{array}{ll}
J u_{x}=\xi_{\psi \psi} \xi_{p p}-\xi_{\psi p}^{2}, & J u_{y}=\xi_{\psi \psi} \eta_{p p}^{\bullet}-\xi_{\psi p} \eta_{\psi p}, \\
J v_{x}=\eta_{\psi \psi} \xi_{p p}-\xi_{\psi p} \eta_{\psi p}, & J v_{y}=\eta_{\psi \psi} \eta_{p p}-\eta_{\psi p}^{2}, \\
J \rho_{x}=\xi_{p p} \rho_{\psi}-\xi_{\psi p} \rho_{p}, & J \rho_{\nu}=\eta_{p p} \rho_{\psi}-\eta_{\psi p} \rho_{p}, \\
J p_{x}=-\xi_{\psi p}, & J p_{\nu}=-\eta_{\psi p},
\end{array}
$$

and the formula

$$
\rho_{p}=\rho^{2}\left(\xi_{\psi p}^{2}+\eta_{\psi p}^{2}-\xi_{\psi \psi} \xi_{p p}-\eta_{\psi \psi} \eta_{p p}\right),
$$

obtained by differentiating (11) partially with respect to $p$, $\psi$. On substituting in (1) from (14), it follows from (4), (11), and (15) that (2) is a solution of (1).

When (13) and the second equation in (11) are solved simultaneously for $\xi_{p p}, \eta_{p p}$, it is found that

$$
\xi_{p p}=\rho J \eta_{\psi}, \quad \eta_{p p}=-\rho J \xi_{\downarrow},
$$

and consequently, from (4), that

$$
\rho^{2} q^{2} J=x_{p}^{2}+y_{p}^{2}, \quad q^{2}=u^{2}+v^{2},
$$

so that the condition

$$
x_{p}^{2}+y_{p}^{2}>0 \text {, }
$$

and (13) are equivalent as long as $\rho \neq 0, q \neq 0$. The last condition amounts to requiring that the directional derivative of the pressure along a streamline remains finite.

The above results may be summed up in the theorem.

TheOREM 1. The solution of the underdetermined system (1) and the solution of the determinate system (11), containing the arbitrary function $\rho=\rho(\psi, p)$ are equivalent problems provided the streamlines are not isobars, the directional derivative of the pressure along the streamlines remains finite, the density does not vanish, and the flow has no stagnation points. 
The specification of the density function $\rho=\rho(\psi, p)$ amounts to assuming that along each streamline the density is a function of the pressure alone, the functional dependence being permitted to vary from one streamline to another.

Returning to the first equation in (11), we may use (4) to write it in the form $u u_{p}+$ $v v_{p}+\rho^{-1}=0$. Holding $\psi$ fast, we integrate this equation from $p_{0}$ to $p$ to obtain Bernoulle's theorem

$$
\frac{1}{2} q^{2}=\frac{1}{2} q_{0}^{2}-\int_{p_{0}}^{p} \rho^{-1} d p, \quad p_{0}=p_{0}(\psi), \quad q_{0}=q_{0}(\psi) .
$$

Here $p_{0}$ is some conveniently chosen reference pressure, not necessarily the same for all streamlines, and $q_{0}$ is the associated fluid velocity, both $p_{0}(\psi), q_{0}(\psi)$ being arbitrary functions.

Thus the system (11) is replaced by the equivalent system (3) in which the fluid velocity $q=q(\psi, p)$, given by Bernoulli's theorem, characterizes the system (3). Apart from the condition $q q_{p} \neq 0$, the function $q$ may be taken arbitrarily, with

$$
\rho=\left(q q_{p}\right)^{-1}, \quad q_{0}=q\left(\psi, p_{0}(\psi)\right) .
$$

On differentiating (17) twice with respect to $p$, it will be found that

$$
M^{2}-1=q q_{p}^{-2} q_{p p},
$$

where $M=q / G$ is the Mach number. The flow is accordingly supersonic or subsonic as $q_{p p}>0$ or $q_{p p}<0$.

For $p_{0}=$ const and a density function of the form $\rho=f(p) / \Psi(\psi)$ we find

$$
\frac{1}{2} q^{2}=\frac{1}{2} q_{0}^{2}-\Psi(\psi) P(p), \quad q_{0}=q_{0}(\psi), \quad P(p)=\int_{p_{0}}^{p} \frac{d p}{f(p)} .
$$

In the special case of a perfect $\operatorname{gas} f(p)=p^{n}, \Psi(\psi)=k^{n}$ with $0<n<1$, we put $p_{0} \equiv 0$ and find

$$
\frac{1}{2} q^{2}=\frac{1}{2} q_{0}^{2}-\frac{k^{n}}{1-n} p^{1-n} .
$$

Here $\frac{1}{2} q_{0}^{2}$ is the total head, $k=k(\psi)=\exp \left(S / c_{v}\right)$, with $S$ denoting the entropy and $c_{v}$ the specific heat at constant volume. For an incompressible fluid with variable density $f(p) \equiv 1$ and

$$
\frac{1}{2} q^{2}=\frac{1}{2} q_{0}^{2}-\Psi(\psi)\left(p-p_{0}\right)
$$

is a linear function of $p$.

The formulae (14) lead to an interesting expression for the vorticity $\omega=u_{y}-v_{x}$. Direct substitution from them, followed by the elimination of $\xi_{p p}, \eta_{p p}$ by (16), leads to

$$
\omega=\frac{q_{\psi}}{q_{p}}=-\rho q q_{\psi} .
$$

For a perfect gas with uniform total head this reduces to

$$
\omega=\frac{n}{1-n} \frac{d S}{d \psi} \frac{p}{c_{v}}
$$


to imply a theorem due to Crocco, ${ }^{7}$ which states that the vorticity is proportional to the pressure along a streamline.

The expressions for the lift and drag functions, given in (4), follow at once from (8), in view of the first column in (4).

3. The exceptional case $p=p(\psi)$. As has been pointed out above, the flows for which the streamlines are isobars must be treated separately. According to Prim, ${ }^{8}$ in the case of a perfect gas, the streamlines for such flows are either concentric circles or parallel straight lines. We shall show how a modification of our previous analysis generalizes Prim's result to any fluid for which the density is a function of the pressure along a streamline.

Under this assumption, $p=p(\psi)$ implies $\rho=\rho(\psi)$. Let us take $\psi, s$ for independent variables, where $s$ denotes the arc length measured along a streamline from some fixed point upon it. One finds that (9) is replaced by

$$
d \xi=\left(u+y p^{\prime}\right) d \psi, d \eta=\left(v-x p^{\prime}\right) d \psi, \quad d \psi=\rho v d x-\rho u d y, \quad '=d / d \psi .
$$

Thus $\xi, \eta$ are functions of $\psi$ alone and we may write

$$
\xi^{\prime}=u+y p^{\prime}, \quad \eta^{\prime}=v-x p^{\prime}, \quad v x_{\psi}-u y_{\psi}=\rho^{-1}, \quad v x_{s}-u y_{s}=0,
$$

the last two equations coming from the last equation in (21), since $\psi, s$ are independent variables. From the first two equations we find

$$
u_{s}+y_{s} p^{\prime}=0, \quad v_{s}-x_{s} p^{\prime}=0,
$$

which, provided $p^{\prime} \neq 0$, may be used to eliminate $x_{s}, y_{s}$ from the last equation in (22) to obtain $u u_{0}+v v_{\mathbb{2}}=0$, and consequently

$$
u^{2}+v^{2}=q^{2}, \quad q=q(\psi) .
$$

When $u, v$ are eliminated from (24) with the aid of (22), we obtain

$$
\left(x+\frac{\eta^{\prime}}{p^{\prime}}\right)^{2}+\left(y-\frac{\xi^{\prime}}{p^{\prime}}\right)^{2}=\frac{q^{2}}{p^{\prime 2}} \text {. }
$$

This shows that any streamline is a circle with center $\left(-\eta^{\prime} / p^{\prime}, \xi^{\prime} / p^{\prime}\right)$ and radius

$$
r=q / p^{\prime}=r(\psi) .
$$

To prove that the circular streamlines are concentric, we employ the identity

$$
\left(\frac{\xi^{\prime}}{p^{\prime}}\right)^{\prime} u+\left(\frac{\eta^{\prime}}{p^{\prime}}\right)^{\prime} v=q r^{\prime}-\rho^{-1}
$$

Since the right side is a function of $\psi$ alone and $u, v$ depend on $s$, this implies

$$
\frac{\xi^{\prime}}{p^{\prime}}=\text { const., } \quad \frac{\eta^{\prime}}{p^{\prime}}=\text { const., }
$$

${ }^{7}$ Crocco, L., Eine neue Stromfunktion fuer die Erforschung der Bewegung der Gase mit Rotation. Zeits. angew. Math. Mech., 17, 4 (1937).

${ }^{8} \mathrm{R}$. C. Prim, On the equations of plane rotational flow of a perfect gas in natural coordinates and on plane rotational gas flow with constant velocity magnitude or constant vorticity along the streamlines. Naval Ordnance Laboratory Memorandum 9264, (1947) SB Project No. 19.2. 
and, as a corollary, that

$$
q r^{\prime}-\rho^{-1}=0 .
$$

If we write (26), (27) in the form

$$
r \frac{d p}{d r} r^{\prime}=q, \quad \rho q r^{\prime}=1,
$$

and eliminate $r^{\prime}$, we obtain

$$
r \frac{d p}{d r}=\rho q^{2},
$$

from which it is clear that any two of $p, q, \rho$ may be specified functions of $r$, and the third is then uniquely determined, if we except $p$ which is only determined up to an additive constant.

If $p^{\prime} \equiv 0$, it follows from (22) that the streamlines form a family of parallel straight lines. Each streamline is a straight line along which $u=\xi^{\prime}, v=\eta^{\prime}$ are constant. To see that these lines are parallel, let

$$
x=x_{0}+s \cos \theta, \quad y=y_{0}+s \sin \theta,
$$

where $x_{0}, y_{0}, \theta$ depend only on $\psi$, be the parametric equations of the family of streamlines. Substitution in the third equation of (22) yields

$$
q\left(x_{0}^{\prime} \sin \theta-y_{0}^{\prime} \cos \theta\right)-q \theta^{\prime} s=\rho^{-1},
$$

which, since $\rho=\rho(\psi)$, implies

$$
\theta^{\prime}=0, \quad \rho q=\left(x_{0}^{\prime} \sin \theta-y_{0}^{\prime} \cos \theta\right)^{-1},
$$

so that $\theta=\theta_{0}=$ const., as stated, and the Cartesian equation of the family of streamlines is

$$
x \sin \theta_{0}-y \cos \theta_{0}=n,
$$

where $n$ denotes the length of the normal let fall from the origin to a streamline. If we take $x_{0}, y_{0}$ in (28) to be the coordinates of the foot of this normal, we have

$$
n^{\prime}=x_{0}^{\prime} \sin \theta_{0}-y_{0}^{\prime} \cos \theta_{0},
$$

from (30), so that the second equation in (29) reduces to

$$
\rho q n^{\prime}=1 .
$$

The following theorem has accordingly been proved.

Theorem 2. For fluids in which the density is a function of the pressure along a streamline, if the streamlines are isobars, the streamlines are either concentric circles ( $p \neq$ const.), or are parallel straight lines $(p=$ const.). In the first case, any two of $\rho, q, p$ may be prescribed as functions of the radius $r$ to determine the third as a function of $r$. In the second case, $\rho, q$ may be taken as arbitrary functions of the distance $n$ of the straight line from the origin.

4. The Munk-Prim substitution principle. A change of parameter $\psi=\psi\left(\psi^{*}\right)$ along the isobars leaves the form of the system (3) unchanged, the new system being

$$
\xi_{\psi}^{*}+\eta_{\psi^{*}}^{* 2}=q^{*^{2}}, \quad \xi_{\psi}^{*} \xi_{p p}^{*}+\eta_{\psi}^{*} \eta_{p p}^{*}=0,
$$


where

$$
\xi^{*}=\xi\left(\psi\left(\psi^{*}\right), p\right), \quad \eta^{*}=\eta\left(\psi\left(\psi^{*}\right), p\right), \quad q^{*}=\left|\frac{d \psi}{d \psi^{*}}\right| q\left(\psi\left(\psi^{*}\right), p\right) .
$$

From (4) we find

$$
\begin{aligned}
& x^{*}=-\eta_{p}^{*}=-\eta_{p}, \quad u^{*}=\xi_{\psi}^{*}=\frac{d \psi}{d \psi^{*}} \xi_{\psi}, \quad \bar{\xi}^{*}=\xi^{*}-p \xi_{p}^{*}=\bar{\xi}, \\
& y^{*}=\quad \xi_{p}^{*}=\xi_{p}, \quad v^{*}=\eta_{\psi^{*}}^{*}=\frac{d \psi}{d \psi^{*}} \eta_{\psi}, \quad \bar{\eta}^{*}=\eta^{*}-p \eta_{p}^{*}=\bar{\eta},
\end{aligned}
$$

so that the new flow has the same streamlines, pressure distribution, lift and drag functions, as the old system. On the other hand, we have

$$
u^{*}=\frac{d \psi}{d \psi^{*}} u, \quad v^{*}=\frac{d \psi}{d \psi^{*}} v, \quad \rho^{*}=\left(\frac{d \psi}{d \psi^{*}}\right)^{-2} \rho
$$

the last relation being a consequence of Bernoulli's Theorem (17).

Thus we are led to the following substitution principle first formulated by Munk and Prim $^{9}$ for steady three-dimensional flows.

From a given flow, a new flow having the same streamlines and pressure distribution may be derived by multiplying the magnitudes of the velocity vectors tangent to a given streamline by the same factor $\lambda$ and the density by the factor $\lambda^{-2}$, the factor $\lambda$ being permitted to change from streamline to streamline.

By properly choosing the factor $\lambda$ either $q_{0}(\psi)$ or $\Psi(\psi)$ in $\left(17^{\prime}\right)$ may be reduced to a constant. Thus, as Munk and Prim have pointed out, in the case of a perfect gas the new flow may be taken to have either constant total head or constant entropy throughout.

5. The Monge-Ampère partial differential equation. The problem of integrating (3) is equivalent to the solution of a Monge-Ampère partial differential equation. To see this, we solve (3) simultaneously for $\eta_{\psi}, \eta_{p p}$ obtaining

$$
\eta_{\psi}=\left(q^{2}-\xi_{\psi}^{2}\right)^{1 / 2}, \quad \eta_{p p}=-\frac{\xi_{\psi} \xi_{p p}}{\left(q^{2}-\xi_{\psi}^{2}\right)^{1 / 2}}
$$

and consequently

$$
\eta_{\psi p}=\frac{q q_{p}-\xi_{\psi} \xi_{\psi p}}{\left(q^{2}-\xi_{\psi}^{2}\right)^{1 / 2}}, \quad \eta_{p p}=-\frac{\xi_{\psi} \xi_{p p}}{\left(q^{2}-\xi_{\psi}^{2}\right)^{1 / 2}}
$$

from which a Monge-Ampère partial differential equation for $\xi=\xi(\psi, p)$

$$
2 q q_{p} \xi_{\psi} \xi_{\psi p}-q q_{\psi} \xi_{\psi} \xi_{p p}+q^{3} q_{p p}-\left(q q_{p p}+q_{p}^{2}\right) \xi_{\psi}^{2}+q^{2}\left(\xi_{\psi \psi} \xi_{p p}-\xi_{\psi p}^{2}\right)=0,
$$

arises when $\eta$ is eliminated by partial differentiation.

Given a solution $\xi$ of (33), we determine $\eta_{p}$ up to an arbitrary constant by the line integral

$$
\eta_{p}=\int\left\{\eta_{p \psi} d \psi+\eta_{p p} d p\right\}
$$

${ }^{9}$ Loc. cit. 
with the aid of (32), and then determine $\eta$ by the line integral

$$
\eta=\int\left\{\eta_{\psi} d \psi+\eta_{p} d p\right\}
$$

with the help of (31), (34). From the symmetry of (3) it is clear that $\eta$ is also a solution of (33).

We propose to investigate the existence and uniqueness of flows when the fluid velocity $q$ in (33) is a prescribed function of $\psi, p$. From (18) this amounts to prescribing the associated fluid velocity $q_{0}=q_{0}(\psi)$ and the density function $\rho=\rho(\psi, p)$.

Consider an arc

$$
C: \quad x=x(p), y=y(p), \quad p_{0} \leqq p \leqq p_{1},
$$

in the physical plane. Can this arc be taken to be one of the streamlines of a flow in which the pressure of the fuid at the point $(x(p), y(p))$ equals the parameter $p$ ? Furthermore, if such a flow exists, is it uniquely determined?

For $C$ to be an arc of a streamline as required, it is necessary and sufficient, from (4), that a constant $\psi_{0}$ exist such that

$$
\xi_{p}\left(\psi_{0}, p\right)=y(p), \quad \xi_{\psi}\left(\psi_{0}, p\right)=q\left(\psi_{0}, p\right) \cos \theta(p),
$$

where

$$
\theta=\arctan y^{\prime} / x^{\prime}, \quad,=d / d p,
$$

is the angle of elevation of the tangent line to $C$. If (35) is replaced by

$$
\xi^{\prime}\left(\psi_{0}, p\right)=c+\int_{p_{0}}^{p} y(p) d p, \quad \xi_{\psi}\left(\psi_{0}, p\right)=q\left(\psi_{0}, p\right) \cos \theta(p), \quad c=\text { const. }
$$

these conditions give the initial data for Cauchy's problem for the partial differential equation (33). To apply the Cauchy-Kowalewski existence theorem, ${ }^{10}$ we write (33) in the form

$$
\xi_{\psi \psi}=\left[q^{2} \xi_{\psi p}^{2}-2 q q_{p} \xi_{\psi} \xi_{\psi p}+q q_{\psi} \xi_{\psi} \xi_{p p}-q^{3} q_{p p}+\left(q q_{p p}+q_{p}^{2}\right) \xi_{\psi}^{2}\right]\left(q^{2} \xi_{p p}\right)^{-1} .
$$

It follows from this theorem that if $q(\psi, p)$ is regular analytic for $\left|\psi-\psi_{0}\right|<\delta, p_{0} \leqq$ $p \leqq p_{1}$ and if $C$ is an analytic arc with $x^{\prime 2}+y^{\prime 2}>0$ holding for $p_{0} \leqq p \leqq p_{1}$, there exists exactly one analytic solution $\xi$ of (37) meeting the initial conditions (36), provided that

$$
q\left(\psi_{0}, p\right) \neq 0, \quad \xi_{p p}\left(\psi_{0}, p\right)=y^{\prime} \neq 0 \quad \text { for } \quad p_{0} \leqq p \leqq p_{1} .
$$

This solution permits the expansion

$$
\xi=h_{0}+h_{1}\left(\psi-\psi_{0}\right)+\cdots+h_{n}\left(\psi-\psi_{0}\right)^{n}+\cdots,\left|\psi-\psi_{0}\right|<\delta_{1}<\delta,
$$

valid for sufficiently small $\delta_{1}$ in which $h_{n}=h_{n}(p)$ are regular analytic functions of $p$ for $p_{0} \leqq p \leqq p_{1}$.

The function $\eta(\psi, p)$ is now determined by (34) after substituting from (39) for $\xi$ in (32), the arbitrary constant in (34) being adjusted so that $\eta_{p}\left(\psi_{0}, p_{0}\right)=-x\left(p_{0}\right)$.

${ }^{10}$ Hadamard, J., Lectures on Cauchy's problem in linear partial differential equations, New Haven (1923) pp. 9-16, in particular p. 16. 
The restriction $y^{\prime} \neq 0$ in (38) is not an essential one. If per chance $y^{\prime}=0$ at some point of $C$, the rôles of $\xi, \eta$ are interchanged in the analysis and (38) replaced by

$$
q\left(\psi_{0}, p\right) \neq 0, \quad \eta_{p D}\left(\psi_{0}, p\right)=-x^{\prime} \neq 0 .
$$

By hypothesis $x^{\prime}, y^{\prime}$ do not vanish simultaneously on $C$, so if (38) fails, we can always be assured that (40) holds.

The condition $q\left(\psi_{0}, p\right) \neq 0$ is equivalent to requiring that there be no stagnation points on $C$.

The above results are summarized in the theorem below.

THEOREM 3. If $C: x=x(p), y=y(p)$ is an analytic arc with $x^{\prime 2}+y^{\prime 2}>0$ holding for $p_{0} \leqq p \leqq p_{1}$, if the fluid velocity $q(\psi, p)$ is regular analytic for $\left|\psi-\psi_{0}\right|<\delta, p_{0} \leqq$ $p \leqq p_{1}$ with $q\left(\psi_{0}, p\right) \neq 0$, the arc $C$ is a streamline for exactly one flow (of preassigned sense on C) with pressure $p$ and fluid velocity $q\left(\psi_{0}, p\right)$ at the point $(x(p), y(p))$. The streamlines and the hodographs of the flow in which $C$ is imbedded are presented parametrically by (4) in which the functions $\xi(\psi, p), \eta(\psi, p)$ permit the expansions (5) valid for sufficiently small $\left|\psi-\psi_{0}\right|$, the coefficients $h, k$ being regular analytic functions of $p$ in the interval $p_{0} \leqq p \leqq p_{1}$.

To calculate the coefficients in (5), these expansions are substituted in (3). On the assumption

$$
q^{2}=q_{0}+q_{1}\left(\psi-\psi_{0}\right)+\cdots, \quad q_{0} \neq 0,
$$

where $q_{0}, q_{1} \cdots$ are regular analytic functions of $p$ for $p_{0} \leqq p \leqq p_{1}$, a routine computatation shows that the $h, k$ are determined by

$$
\begin{aligned}
& \sum_{r=0}^{n}(r+1)(n+1-r)\left(h_{r+1} h_{n+1-r}+k_{r+1} k_{n+1-r}\right)=q_{n}, \\
& \sum_{r=0}^{n}(r+1)\left(h_{n-r}^{\prime \prime} h_{r+1}+k_{n-r}^{\prime \prime} k_{r+1}\right)=0 .
\end{aligned}
$$

In particular, for $n=0,1,2$, we find

$$
\begin{aligned}
h_{1}^{2}+k_{1}^{2} & =q_{0}, \quad h_{0}^{\prime \prime} h_{1}+k_{0}^{\prime \prime} k_{1}=0, \\
h_{1} h_{2}+k_{1} k_{2} & =\frac{q_{1}}{4}, \quad h_{0}^{\prime \prime} h_{2}+k_{0}^{\prime \prime} k_{2}=-\frac{1}{2}\left(h_{1}^{\prime \prime} h_{1}+k_{1}^{\prime \prime} k_{1}\right), \\
h_{1} h_{3}+k_{1} k_{3} & =\frac{q_{2}}{6}-\frac{2}{3}\left(h_{2}^{2}+k_{2}^{2}\right), \\
h_{0}^{\prime \prime} h_{3}+k_{0}^{\prime \prime} k_{3} & =-\frac{1}{3}\left(h_{2}^{\prime \prime} h_{1}+k_{2}^{\prime \prime} k_{1}\right)-\frac{2}{3}\left(h_{1}^{\prime \prime} h_{2}+k_{1}^{\prime \prime} k_{2}\right) .
\end{aligned}
$$

The coefficients $h_{0}, k_{0}$ are, from (4), determined by

$$
h_{0}^{\prime}=y(p), \quad k_{0}^{\prime}=-x(p) .
$$

The first pair of equations in (43) are solved simultaneously to determine $h_{1}, k_{1}$ and the second pair may then be solved simultaneously for $h_{2}, k_{2}$, since the determinant

$$
h_{1} k_{0}^{\prime \prime}-k_{1} h_{0}^{\prime \prime}=\left[q_{0}\left(x^{\prime 2}+y^{\prime 2}\right)\right]^{1 / 2},
$$


does not vanish by hypothesis. Proceeding in this manner, the remaining coefficients are determined step by step.

6. Prandtl-Meyer flows. Leaving aside the case where the density or velocity vanishes, it is clear from (20) that a necessary and sufficient condition for the flow to be irrotational is $q_{\psi}=0$.

For (3) to have a solution (5)

$$
\xi=h_{0}+h_{1}\left(\psi-\psi_{0}\right), \quad \eta=k_{0}+k_{1}\left(\psi-\psi_{0}\right),
$$

linear in $\psi-\psi_{0}$, it is clearly necessary that $q_{\psi}=0$, that is, that the flow be irrotational. Conversely, we shall now show that among the irrotational flows there are solutions (44) of (3).

We set

$$
q=q(p)
$$

in (3) and take $q_{n-1}=h_{n}=k_{n}=0$ for $n=2,3, \cdots$ in (42), or (43), to get following set

$$
h_{1}^{2}+k_{1}^{2}=q^{2}, \quad h_{1} h_{1}^{\prime \prime}+k_{1} k_{1}^{\prime \prime}=0, \quad h_{1} h_{0}^{\prime \prime}+k_{1} k_{0}^{\prime \prime}=0,
$$

of necessary and sufficient conditions for (44) to be a solution of (3). To satisfy the first pair of equations, we place

$$
h_{1}=q \cos \theta, \quad k_{1}=q \sin \theta,
$$

and find that

$$
\theta=\theta(p)=\theta_{0}+\int_{p_{0}}^{p}\left(\frac{q^{\prime \prime}}{q}\right)^{1 / 2} d p
$$

to determine $h_{1}, k_{1}$ in (46) as functions of $p$.

Leaving aside, for the moment, the determination of $h_{0}, k_{0}$, we substitute in (4) from (44) to obtain

$$
\begin{array}{ll}
x=-k_{0}^{\prime}-s^{\prime}\left(\psi-\psi_{0}\right) \cos (\theta-\mu), & u=q(p) \cos \theta(p)=u(p), \\
y=h_{0}^{\prime}-s^{\prime}\left(\psi-\psi_{0}\right) \sin (\theta-\mu), & v=q(p) \sin \theta(p)=v(p),
\end{array}
$$

where $q(p), \theta(p)$ are the functions of $p$ mentioned in (45), (48), and $s$ denotes the arc length of the hodograph curve

$$
H: \quad u=u(p), \quad v=v(p),
$$

in the hodograph plane. The angle $\mu$ defined by

$$
\cot \mu=q \theta^{\prime} / q^{\prime}
$$

is the angle between the radius vector $O P$ to a point $P$ of $H$ and the perpendicular $O N$ let fall on the tangent line to $H$ at $P$ (see figure below). From (48) and (19) we find

$$
\cot \mu=\left(M^{2}-1\right)^{1 / 2}, \quad s^{\prime}=s^{\prime}(p)=-(G \rho)^{-1},
$$

so that $\mu=\mu(p)$ is identified as the Mach angle.

On placing $p=$ const. in (49), it is clear that the isobars are straight lines along which all velocity vectors have the same magnitude and make the same angle $\mu$ with the isobar. These straight lines are the "Mach lines" in the flow defined by (49). 
With $\psi=$ const. in (49), the streamlines are presented parametrically in terms of the pressure $p$ by the equations in the first column. From the second column it is clear that all streamlines have the same hodograph, a characteristic feature of Prandtl-Meyer flows.

We return to the determination of $h_{0}, k_{0}$. Taking $\psi=\psi_{0}$ in (49), we obtain an initial streamline

$$
x=x(p)=-k_{0}^{\prime}, \quad y=y(p)=h_{0}^{\prime},
$$

in terms of which the last condition in (46) becomes

$$
y^{\prime} \cos \theta-x^{\prime} \sin \theta=0,
$$

on substituting from (47). Thus we may take any curve

$$
C: \quad x=f(\tau), \quad y=g(\tau), \quad\left(\frac{d f}{d \tau}\right)^{2}+\left(\frac{d g}{d \tau}\right)^{2}>0,
$$

with continuous non vanishing curvature for the initial streamline, for if we write

$$
x(p)=f(\tau), \quad y(p)=g(\tau), \quad \tau=\tau(p)
$$

and introduce the slope $m=m(\tau)$ of $C$, condition (52) becomes

$$
m(\tau)=\tan \theta(p)
$$

an implicit equation for the determination of the parameterization $\tau=\tau(p)$. In other words, once $C$ is given, the pressure distribution along it is determined by (53).

The above considerations are illuminated by the following geometrical construction.
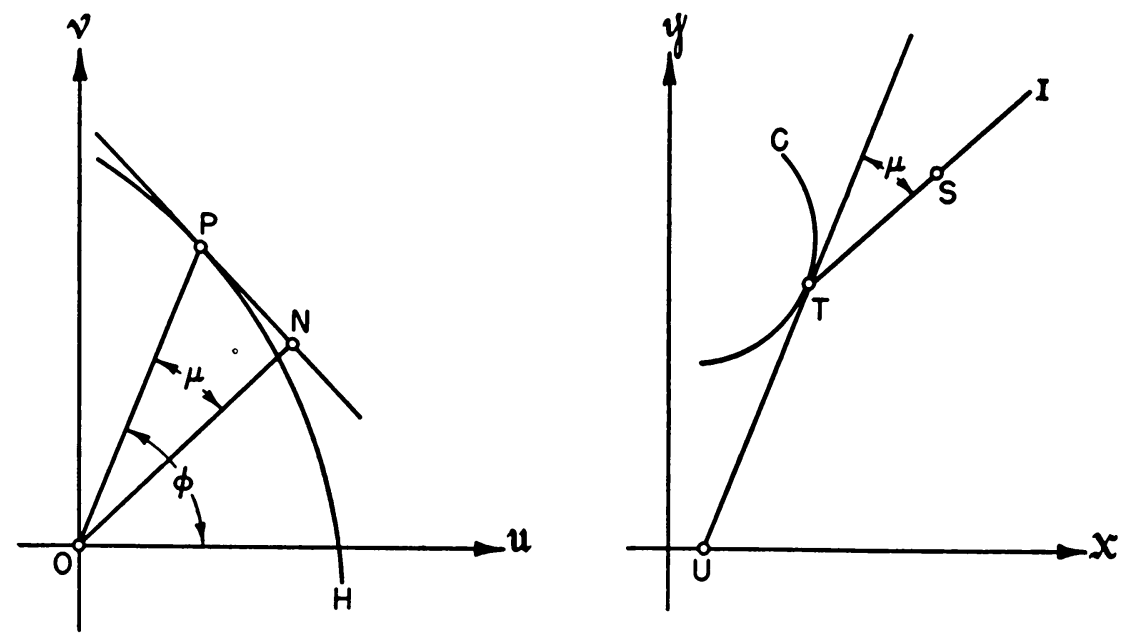

FIG. 1.

At a point $T$ with parameter $\tau$ on $C$ in the physical plane we draw the tangent line $T U$. From $O$ draw $O P$ in the hodograph plane parallel to $T U$ to meet the hodograph curve $H$ at $P$ with parameter $p$. This determines geometrically the correspondence $\tau=\tau(p)$ between the parameter $\tau$ at $T$ and the parameter $p$ at $P$ affected analytically by $(53)$. 
The isobar through $T$ is constructed by drawing a line $T I$ parallel to the normal $O N$ let fall upon the tangent line to $H$ at $P$. To construct a streamline, the length $(G \rho)^{-1}$ is laid off from $T$ along $T I$ to determine a point $S$. The locus of $S$ as $T$ traces out $C$ is a streamline. The remaining streamlines are "homothetic transformations" of this streamline, obtained by laying off the lengths $\left(\psi-\psi_{0}\right)(\rho G)^{-1}$ along $T I$ from $T$.

7. The drag and lift functions. The Monge-Ampère equation (33) is carried over into a quasi-linear equation

$q^{2} \bar{\xi}_{\psi \psi}+2 q q_{p} \bar{\xi}_{\psi} \bar{\xi}_{\psi \nu}-\left[q^{3} q_{p D}-\left(q q_{p D}+q_{p}^{2}\right) \bar{\xi}_{\psi}^{2}\right] \bar{\xi}_{\nu \nu}-q q_{\psi} \bar{\xi}_{\psi}=0, \quad q=q\left(\psi,-\bar{\xi}_{\nu}\right)$,

with $\psi, y$ in place of $\psi, p$ as independent variables, and the drag function $\bar{\xi}=\bar{\xi}(\psi, y)$ as unknown function, by the Ampère contact transformation

$$
\bar{\psi}=\psi, \quad \bar{p}=y, \quad \bar{\xi}=\xi-y p, \quad \bar{u}=u, \quad \bar{y}=-p .
$$

With $\psi, y$ serving as independent variables, (7) is written

$$
d \bar{\xi}=u d \psi-p d y, \quad d \psi=\rho v x_{\psi} d \psi+\rho\left(v x_{\nu}-u\right) d y,
$$

from which we see that

$$
u=\bar{\xi}_{\psi}, \quad p=-\xi_{\nu}, \quad x_{\psi}=(\rho v)^{-1}, \quad x_{\nu}=u v^{-1} .
$$

Since $u^{2}+v^{2}=q^{2}$, the last two equations may, from (18), be written

$$
x_{\psi}=-\frac{q q_{p}}{\left(q^{2}-\bar{\xi}_{\psi}^{2}\right)^{1 / 2}}, \quad x_{\nu}=\frac{\bar{\xi}_{\psi}}{\left(q^{2}-\bar{\xi}_{\psi}^{2}\right)^{1 / 2}},
$$

from which $x$ may be eliminated to obtain a second derivation of (54).

Here $p$ is presumed to be replaced by $-\bar{\xi}_{y}$. Given a solution $\bar{\xi}=\bar{\xi}(\psi, y)$ of $(54)$, the Cartesian equation of the streamlines is

$$
x=x(\psi, y)=\int\left\{x_{\psi} d \psi+x_{\nu} d y\right\},
$$

where $x_{\psi}, x_{\nu}$ are the functions of $\psi, y$ given in (55). The pressure $p$ and velocity components are, of course, given by

$$
p=-\bar{\xi}_{\nu}, \quad u=\bar{\xi}_{\psi}, \quad v=\left(q^{2}-\bar{\xi}_{\psi}^{2}\right)^{1 / 2} .
$$

Formulas analogous to the above have been obtained by Kiebel with $-\psi, x$ as independent variables for the special case of a perfect gas, without, however, explicitly computing (54) or noticing the physical significance of $\bar{\xi}$. To obtain the actual formulas of Kiebel, the lift function $\bar{\eta}$ is used in place of the drag function $\bar{\xi}$, the lift function $\bar{\eta}$ becoming the function ${ }^{11} \chi$ used by Kiebel in his investigation of flows for which $\chi$ has the special form

$$
\chi=-H(\psi) x^{-(\gamma-1) /(\gamma+1)} .
$$

8. Unsteady one-dimensional flow. If we disregard the second equation in (1) and set

$$
v \equiv 1, \quad y \equiv t,
$$

${ }^{11} O p$. cit. p. 2. 
in the remaining equations, we obtain the differential equations

$$
\rho\left(u_{x} u+u_{t}\right)+p_{x}=0, \quad(\rho u)_{x}+\rho_{t}=0,
$$

for unsteady one-dimensional flow. We find (9) replaced by

$$
d \xi=u d \psi+t d p, \quad d \psi=\rho d x-\rho u d t,
$$

and taking $\psi, p$ for independent variables, ${ }^{12}$ we find, instead of (11)

$$
\xi_{\psi} \xi_{\psi p}-x_{\psi}+\rho^{-1}=0, \quad \xi_{\psi} \xi_{p p}-x_{p}=0,
$$

where $\rho=\rho(\psi, p)$ is an arbitrary function. Eliminating $x$ by partial differentiation, the rôle of (33) is taken over by

$$
\xi_{\psi \downarrow} \xi_{p p}-\xi_{\psi p}^{2}=\left(\frac{1}{\rho}\right)_{p},
$$

a differential equation which was obtained by another method by the author ${ }^{13}$ in the special case of a perfect gas.

Given a solution $\xi=\xi(\psi, p)$ of (58), the flow is presented by

$$
x=x(\psi, p)=\int\left\{\left(\xi_{\psi} \xi_{\psi_{p}}+\rho^{-1}\right) d \psi+\xi_{\psi} \xi_{p p} d p\right\}, \quad u=\xi_{\psi}(\psi, p), \quad t=\xi_{p}(\psi, p),
$$

in view of (56), (57); or, provided $\xi_{p p} \neq 0$, so the last equation can be solved for $p$, the equations defining the flow can be put in the form

$$
x=x(\psi, t), \quad u=u(\psi, t), \quad p=p(\psi, t) .
$$

For fixed $\psi$ these equations give the positions $x$, velocity $u$, and pressure $p$ for a gas particle as functions of the time $t$.

12The case $p=p(\psi)$ is disregarded.

${ }^{13}$ Martin, M. H., The rectilinear motion of a gas II, Amér. J. Math. 57, 410 (1945). 